\title{
Genome-wide exploration of the molecular evolution and regulatory network of mitogen-activated protein kinase cascades upon multiple stresses in Brachypodium distachyon
}

\author{
Min Jiang ${ }^{1+}$, Feng Wen ${ }^{1,2+}$, Jianmei Cao ${ }^{1}$, Peng $\mathrm{Li}^{1}$, Jessica She ${ }^{1,3}$ and Zhaoging Chu ${ }^{1 *}$
}

\begin{abstract}
Background: Brachypodium distachyon is emerging as a widely recognized model plant that has very close relations with several economically important Poaceae species. MAPK cascade is known to be an evolutionarily conserved signaling module involved in multiple stresses. Although the gene sequences of MAPK and MAPKK family have been fully identified in $B$. distachyon, the information related to the upstream MAPKKK gene family especially the regulatory network among MAPKs, MAPKKs and MAPKKKs upon multiple stresses remains to be understood.

Results: In this study, we have identified MAPKKKs which belong to the biggest gene family of MAPK cascade kinases. We have systematically investigated the evolution of whole MAPK cascade kinase gene family in terms of gene structures, protein structural organization, chromosomal localization, orthologs construction and gene duplication analysis. Our results showed that most BdMAPK cascade kinases were located at the low-CpG-density region, and the clustered members in each group shared similar structures of the genes and proteins. Synteny analysis showed that 62 or 21 pairs of duplicated orthologs were present between B. distachyon and Oryza sativa, or between B. distachyon and Arabidopsis thaliana respectively. Gene expression data revealed that BdMAPK cascade kinases were rapidly regulated by stresses and phytohormones. Importantly, we have constructed a regulation network based on co-expression patterns of the expression profiles upon multiple stresses performed in this study.

Conclusions: BdMAPK cascade kinases were involved in the signaling pathways of multiple stresses in B. distachyon. The network of co-expression regulation showed the most of duplicated BdMAPK cascade kinase gene orthologs demonstrated their convergent function, whereas few of them developed divergent function in the evolutionary process. The molecular evolution analysis of identified MAPK family genes and the constructed MAPK cascade regulation network under multiple stresses provide valuable information for further investigation of the functions of BdMAPK cascade kinase genes.
\end{abstract}

Keywords: MAPK cascade kinases, Brachypodium distachyon, Evolution, Gene expression, Abiotic and biotic stresses

\footnotetext{
* Correspondence: zqchu@sibs.ac.cn

${ }^{\dagger}$ Equal contributors

'Shanghai Chenshan Plant Science Research Center, Shanghai Chenshan

Botanical Garden, Shanghai Key Laboratory of Plant Functional Genomics

and Resources, Shanghai Institutes for Biological Sciences, Chinese Academy

of Sciences, 3888 Chenhua Road, 201602 Shanghai, Songjiang, China

Full list of author information is available at the end of the article
}

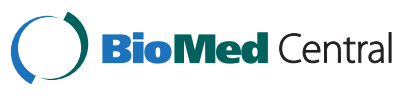

(c) 2015 Jiang et al.; licensee BioMed Central. This is an Open Access article distributed under the terms of the Creative Commons Attribution License (http://creativecommons.org/licenses/by/4.0), which permits unrestricted use, distribution, and reproduction in any medium, provided the original work is properly credited. The Creative Commons Public Domain Dedication waiver (http://creativecommons.org/publicdomain/zero/1.0/) applies to the data made available in this article unless otherwise stated. 


\section{Background}

Brachypodium distachyon is emerging as a widely recognized model plant of the grass subfamily Pooideae whose genome is completely sequenced [1]. The plant has a very close relationship with several economically important Poaceae species such as Oryza sativa, Sorghum bicolor, Triticum aestivum and turf grasses. The study of the $B$. distachyon genome will help scientists better understand the mechanism of gene-controlled physiological processes in Poaceae, and subsequently improve the abiotic or biotic stress tolerance of crops and turf grasses through gene engineering.

Mitogen-activated protein kinases (MAPKs) cascades are evolutionarily conserved signaling modules, which are involved in controlling many cellular functions, including cell division, development and multiple stresses in all eukaryotes [2-4]. Activated MAPK cascade kinases can regulate the phosphorylation level of transcription factors and other components related to the MAPK pathway. For example, reactive oxygen species (ROS) can active MEKK1-MPK4 cascade, and the activated MAPK cascade further regulates the ROS-responsive gene expression and other MAPKs [5]. Previous findings indicated that the MAPK cascade of MEKK1-MKK4/MKK5-MPK3/MPK6 was responsible for signal transmission when a plant recognized flagellin, and then triggered the disease resistant responses [6,7]. The MAPK cascade is not only involved in stresses responses, but also plays important roles in response to phytohormone. For instance, the MAPK cascade of MKK3-MPK6 has been proved as an important part of jasmonic acid (JA) signal transduction pathway in Arabidopsis thaliana [8,9]. Generally, a MAPK cascade contains three functionally conserved components: MAPKs, MAPK Kinases (MAPKKs/MKKs) and MAPKK Kinases (MAPKKKs/MEKKs). The external stimuli, once perceived by a membrane receptor, could translate them into cellular response signals, resulting in subsequent phosphorylation of MAPKKKs. The kinaseactivation process of MAPKKKs initiates with the activation of MAPKKs by phosphorylating the serine and threonine residues in the S/TXXXXXS/T motif, then MAPKs at the last step of MAPK cascade through phosphorylation of both tyrosine and threonine residues in the TXY motif $[4,10]$.

To date, numerous studies on plant MAPKs (also called MPKs) were conducted by many scientists [11]. Similar to animal ERK kinases, plants MAPKs also have two phosphorylation motifs of TDY and TEY. A. thaliana MPKs, which have been reported to classify into four groups, are involved in developmental processes and the activation in response to biotic and abiotic stresses [12-14]. In A. thaliana, the groups of A and B including AtMPK3, AtMPK4 and AtMPK6 were wellcharacterized to respond to a diversity of environmental stimuli. For example, AtMPK4 was activated in a few minutes in response to the flg22 peptide of flagellin, and the activated AtMPK4 controlled the disease resistance gene expression and defense responses, showing a negative regulation in the biotic stress treatment $[6,15]$. AtMPK3 and AtMPK6 were involved in various environmental stress and hormone responses. Studies also revealed that MPK3 and MPK6 were activated when $A$. thaliana seedlings were treated with flg22. MPK3 and MPK6 were proved to be involved in other signaling pathways which are independent of MPK4 [6,7]. So far, ten MAPKKs in $A$. thaliana and eight MAPKKs in $O$. sativa have been found in response to multiple stresses. The upstream activation of AtMPK4 by AtMKK1 and AtMKK2 were involved in not only the ROS homeostasis and salicylic acid (SA) accumulation, but also abiotic stresses such as cold, salt and wounding [16-18]. OsMEK1, a homolog of AtMKK1, has been demonstrated to play an important role in response to the low-temperature stress at wide ranges in O. sativa [19]. MAPKKKs (also named as MAP3Ks and MEKKs), known as the first step of MAPK cascade, constitute a diverse family of kinases which have been grouped into three large subfamilies based on the sequence of the kinase catalytic domain: the MEKK-like family, Raf-like family and ZIK-like family. Similar to mammalian MEKK1 and yeast STE11 and BCK1, plant MEKK-like subfamily members with a conserved catalytic domain are involved in stress responses by activating downstream MKKs [20]. In A. thaliana, AtMEKK1, as a downstream kinase of the flagellin receptor FLS2, can trigger a complete plant MAPK cascade (MEKK1, MKK4/MKK5 and MPK3/MPK6), then function as a conservation innate immunity in response to both bacterial and fungal pathogens [7]. The Raf-like family possesses more than half of MAPKKKs members, and the subfamily members share a specific polypeptide signature GTxx (W/Y) MAPE, which are similar to mammalian RAF1 $[12,21,22]$. The A. thaliana Raf-like members, Enhanced Disease Resistance 1 (EDR1) and Constitutive Triple Response 1 (CTR1), shared a homology with mammalian Raf-like MAPKKKs, were reported to participate in ethylene-mediated signaling and defense responses [23-26]. Sister clades such as ZIK-like kinases are presented in MAPKKKs phylogenetic analyses, however, these enzymes have not been shown to phosphorylate MAPKKs in plants [20,27].

So far, many members of MAPK cascades have been identified using functional genomic methods. Twenty MAPKs and 10 MAPKKs have been found in $A$. thaliana, 15 MAPKs and 8 MAPKKs are present in the $O$. sativa genome, while 16 MAPK genes and 12 MAPKK genes were identified from $B$. distachyon $\mathrm{Bd} 21$ genome [28]. MAPKKK gene family has been systematically investigated in A. thaliana, O. sativa, Zea mays and Gossypium raimondii [21,22,29-31]. In this study, we have systematically identified all MAPK cascade kinase genes including a 
total of 75 MAPKKK genes from $B$. distachyon $\mathrm{Bd} 21$ genome. We further investigated the evolutionary relationship of $B$. distachyon MAPK cascade kinase genes (MAPKs, MAPKKs and MAPKKKs) in terms of phytogenetic analysis, chromosomal localization and gene duplication with their counterparts from monocot $O$. sativa and $\operatorname{dicot} A$. thaliana. Subsequently, we used qRT-PCR to examine their tissue-specific transcription profiles and the profiles in response to several biotic or abiotic stresses. In addition, we analyzed the expression changes of BdMAPK cascade kinase genes under those treatments, and established the MAPK signaling network based on the co-expression patterns upon different stresses treatment. The duplicated ortholog pairs of BdMAPK cascade kinase genes revealed their convergent or divergent function in the process of evolution. Our study provided the genome-wide evolutionary analyses and expression profiles of $M A P K$ cascade kinase genes in $B$. distachyon under multiple-stress conditions, which paved a way for further investigation into MAPK cascade kinase genes functions across different plant species.

\section{Results and discussion}

Distribution of MAPK cascade kinase genes in the plants

MAPK cascades exist in all eukaryotes and orchestrate diverse cellular activities. Here, we searched for MAPK cascade kinase genes in ten common plant species from the reported data and the Plaza Database (http://bioinformatics. psb.ugent.be/plaza/). The result indicated that these genes play important roles in regulation of many cellular functions in eukaryotes, especially MAPK cascade kinase genes are widely existed in the plants (Figure 1). In general, the number of MAPK family members was more than that of MAPKK gene members. As the first step of the MAPK cascade, MAPKKK genes transmit all kinds of up-stream signals into the MAPK cascade. The MAPKKKs have the largest number among MAPK, MAPKK and MAPKKK gene families. Unfortunately,

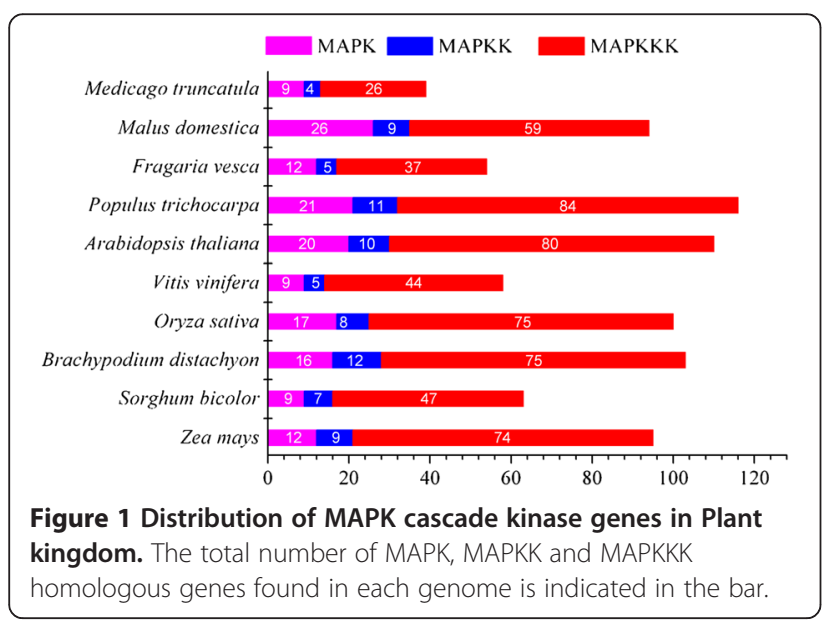

only a few reports about MAPKKK genes have been found in the literature due to its multiformity $[29,30]$. To date, 16 MAPK genes and 12 MAPKK genes, but no report about MAPKKK genes were identified from $B$. distachyon genome [28].

\section{Identification and annotation of the MAPK cascade kinase} gene family from the $B$. distachyon genome

The availability of a completed genome of $B$. distachyon offers the feasibility to identify all the MAPK cascade kinase gene family members in the plant. The identification and phylogenetic analyses of MAPK and MAPKK genes have been completed in B. distachyon previously, and therefore we used the same annotation of MAPK and MAPKK genes in this study as reported previously (Additional file 1) [28]. In order to identify the MAPKKK genes, 155 queries of the MAPKKK sequences from $A$. thaliana and $O$. sativa were employed for BLASTP analyses against 32255 sequences of the protein database of $B$. distachyon available from MIPS (http://mips.helmholtzmuenchen.de/plant/brachypodium/), which retrieved 163 hits as target sequences [1]. A self BLAST of these sequences followed by manual editing to remove the redundancy has finally identified 75 MAPKKK genes from the $B$. distachyon genome (Additional file 1). Furthermore, we analyzed the sequence homology between putative $B d M A P K K K$ genes and the MAPKKK gene family in $O$. sativa using the Best Blast Mutual Hit (BBMH) method. Since there was no nomenclature rule of MAPKKKS to follow in A. thaliana and O. sativa, all 75 MAPKKK gene family members in $B$. distachyon genome were designated as BdMAPKKK1-BdMAPKKK75 based on the BBMH scores. The amino acid sequence analyses showed that all 75 BdMAPKKKs have a conserved protein kinase domain in the MAPK family. Protein subcellular localization was predicted by WoLF PSORT online analysis, and only the maximum probability was selected. The results revealed that most MAPK cascade kinases varied from the cytoplasm, chloroplast, and mitochondria to the nucleus except for 4 MAPKKKs and 4 MAPKs. For example, BdMAPKKK40 and BdMAPKKK75 were present in the cytoskeleton, whereas BdMAPKKK19 was localized in peroxisomes (Additional file 1).

\section{Gene and protein structural organization of BdMAPKs, BdMAPKKs and BdMAPKKKs}

In order to seek the evolutionary relationship among MAPK, MAPKK and MAPKKK family genes and further analyze the regulation network among these family genes, phylogenetic trees were constructed from alignments of the full-length MAPK cascade kinase nucleotide sequences using the Neighbor-Joining (NJ) method by MEGA5.0 (Figure 2A). According to the phylogenetic analyses of the nucleotide sequences, BdMAPKs were 


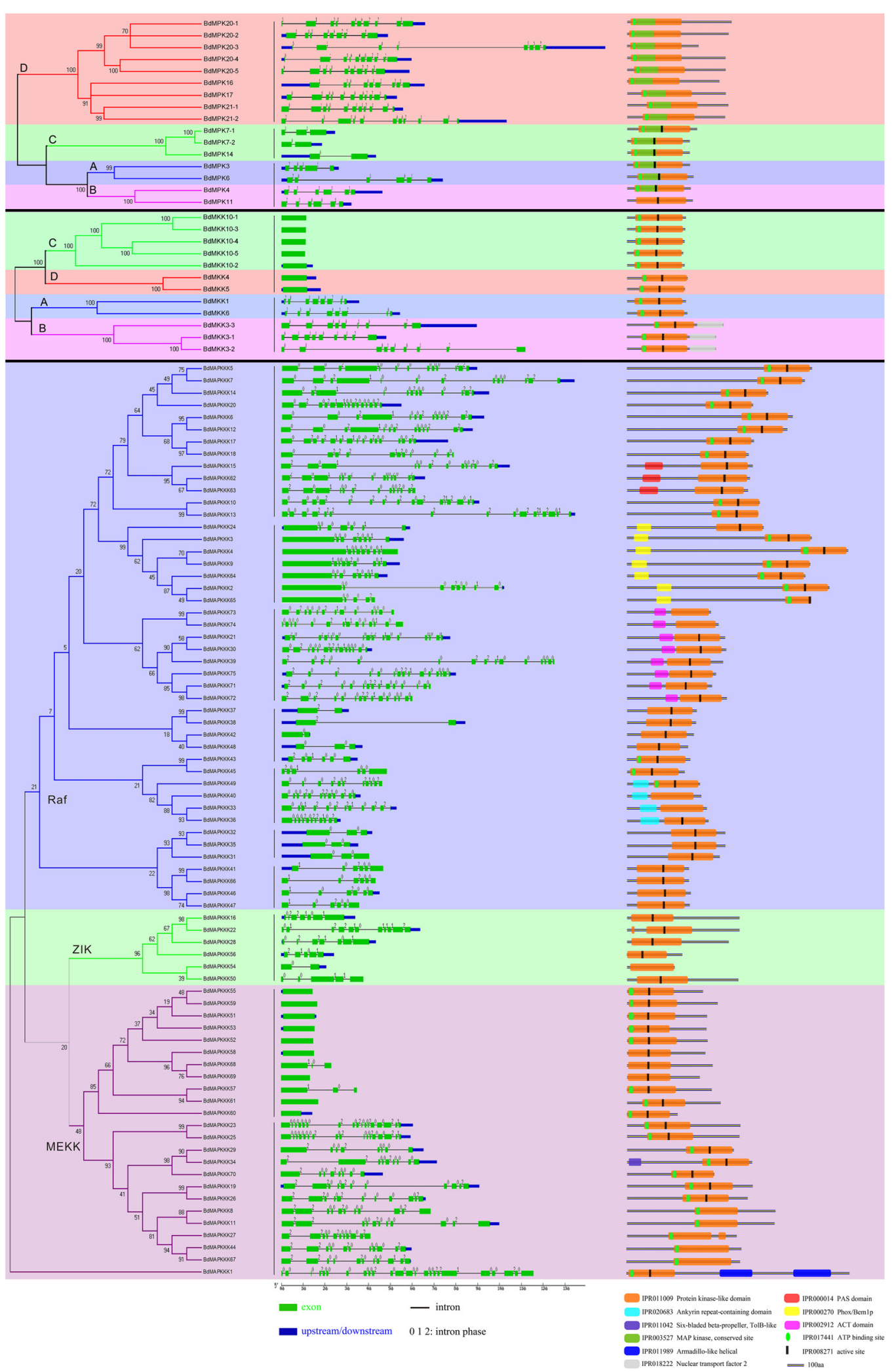

Figure 2 Phylogenetic relationships (A), gene structures (B) and protein structures (C) analysis of MAPK cascade kinases in B. distachyon. 
classified into four groups, consistent with the MAPKs in A. thaliana. Similarly, BdMAPKKs were also divided into four groups. The results were consistent with the previous study [28]. Correspondingly, MAPKKKs were allocated into three groups based on their sequence alignments: the MEKK-like family, Raf-like family and ZIK-like family. To date, there is no report regarding MAPKKK genes in $B$. distachyon, so searching for MAPKKK family genes in $B$. distachyon is necessary. The phylogenetic analysis indicated that BdMAPKKKs can also be divided into three major groups: MEKK, Raf and ZIK. The result showed that the Raf-like family contains large members, while the ZIK-like family only has six BdMAPKKK members.

Next, we analyzed the gene structure, exon position and phases of intron in BdMAPK cascade kinase genes, and 15 kinds of gene structures were identified (Figure 2B). Generally, the MAPK, MAPKK and MAPKKK genes could be divided into 3, 2 and 10 subgroups based on their exon/intron structures, respectively (Figure $2 \mathrm{~B}$ ). As shown in Figure 2B, all the members of the MAPK family had introns, whereas the members of groups $C$ and D in the MAPKK family had no intron. In the Raf-like gene family, the number of introns varied from 1 to 15 , whereas the number of exons varied from 2 to 11 in the ZIK-like gene family. Half of the MEKK group genes had only one exon and no intron. The results showed that the BdMAPK cascade kinase genes were consistent with their homologous genes in A. thaliana, O. sativa and Zea mays by gene structure analyses [22]. Additionally, most gene pairs, which were clustered together by phylogenetic analysis, shared a similar exon/intron structure and intron phases. The conserved exon/intron structure and intron phases in the BdMAPK cascade kinase genes revealed the close evolutionary relationship among all four species, and supported their classification.

Furthermore, we have predicted the protein domains of the BdMAPK cascade kinase family using InterProScan against protein databases. The structure of all members of the BdMAPK cascade kinases was shown as a scheme in Figure 2C. Generally, each cluster of the BdMAPK cascade kinases by phylogenetic analysis shared a similar protein structure. All members of the BdMAPKs, BdMAPKKs and BdMAPKKKs contained a protein kinase domain (IPR011009). Most protein kinases catalyzed the transfer of a phosphate group from nucleoside triphosphates (often ATP) to specific amino acid residues of a protein substrate, resulting in a conformational change affecting protein function. The ATP-binding site, which is located on the N-terminal extremity of the catalytic domain, belongs to the most conserved sequences in the protein kinase family. Thus, most BdMAPKs, BdMAPKKs and BdMAPKKKs contained an ATP-binding site (IPR017441), which suggested that these BdMAPK cascade kinases used
ATP as a ligand in signal transduction pathways. In the central part of the catalytic domain, almost all BdMAPK cascade kinases, except the group D in the BdMAPKs, contain the active site conserved aspartic acid residue (IPR008271), which is important for the catalytic activity of the enzyme. The catalytic domain and their structures in the BdMAPK cascade kinases are similar among proteins within subfamilies, demonstrating that the protein architecture is remarkably conserved within a specific subfamily. Moreover, despite all of BdMAPK cascade kinases contained a protein kinase domain, many of them contained other domains, such as ACT domain, PAS domain, and Ankyrin repeat containing domain. Interestingly, these BdMAPK cascade kinases which contained the same domain were clustered together to a clade, and showed similar expression patterns in response to multiple stresses treatment. For instances, most Phox/Bemlp domain containing BdMAPKKK genes showed to be down-regulated after PEG or $\mathrm{H}_{2} \mathrm{O}_{2}$ treatment, while the ACT domain containing BdMAPKKK genes were up-regulated by $\mathrm{Cd}^{2+}$. These results suggested that the BdMAPK cascade kinases exhibited different biological functions in response to various physiologic reactions. Because the biological functions of many MAPK cascade kinase genes in $B$. distachyon remain to be elucidated, the above findings may facilitate the identification of functional units in BdMAPK cascade kinase genes and lead to the discovery of their roles in plant growth and development.

\section{Genomic distribution and gene duplication of BdMAPKs, BdMAPKKs and BdMAPKKKs}

Although the genome of $B$. distachyon has already been sequenced, the information regarding genomic distribution and gene duplication of the BdMAPK cascade gene family in $B$. distachyon remains unclear. In order to investigate the relationship between genetic divergence and gene duplication within the BdMAPK cascade gene family in $B$. distachyon, we investigated the chromosomal locations of the BdMAPK cascade kinases genes based on the information from the $B$. distachyon genomic database (http://www.brachypodium.org/). The physical locations of the MAPK cascade kinase genes on $B$. distachyon chromosomes and the CPG island distribution map were depicted in Figure 3. We found that 103 BdMAPK cascade kinases were mapped on all 5 chromosomes of $B$. distachyon, in which 35 genes were present on chromosomes 1; 25 genes were located on chromosomes 2; and 25 genes were located on chromosomes 3. In addition, chromosome 4 had 14 MAPK cascade kinases, whereas chromosome 5 only encoded 4 MAPKKKs members. Interestingly, all members of the BdMAPK cascade kinase genes were located at the low density region of $\mathrm{CpG}$ islands on all 5 chromosomes. As shown in Figure 3, 26 pairs of duplication genes were 


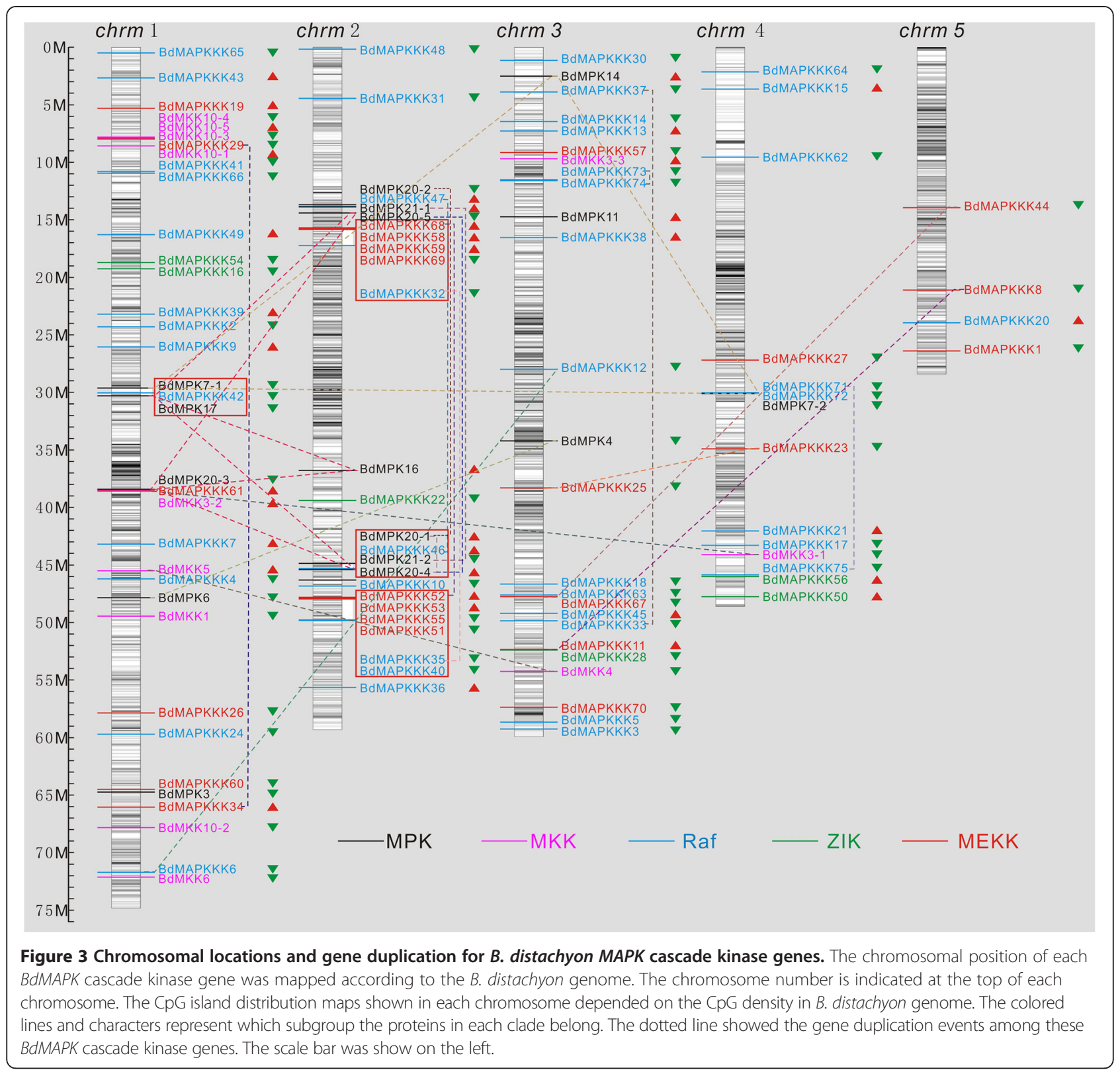

identified, including 11 duplication events within the same chromosome and 15 segmental duplication events between chromosomes. This result suggested the duplication events could play vital roles in the expansion of the MAPK cascade kinase genes in the $B$. distachyon genome. Interestingly, among these paralogs, 8 gene pairs located at chromosome 2, 3 and 5 formed two pairs of duplicated chromosome regions, which were consistent with the result of duplicated regions of $B d W R K Y$ genes as reported previously [32]. These results revealed that members of the large gene family might be the consequence of genomic rearrangements and expansions during the process of evolution. Many DNA fragments from different origins could be recombined into another chromosome to form a new member of the large gene family. The function of the duplicated gene might be diverged by base substitutions, deletions and insertions.

\section{Comparative analysis of the phylogenetic ortholog} genes of MAPKs, MAPKKs and MAPKKKs in B. distachyon,

\section{A. thaliana and $O$. sativa}

To examine the evolutionary relationships between different MAPK cascade kinases in B. distachyon, A. thaliana and $O$. sativa, phylogenetic trees were constructed from alignments of the full MAPK cascade kinase amino acid sequences using the Neighbor-Joining (NJ) method by MEGA5.0 (Additional file 2). The gene model and amino acid sequences of MAPK cascade kinases in $B$. 
distachyon, $A$. thaliana and $O$. sativa were shown in Additional files 3 and 4. The phylogenetic analysis indicated that each of the BdMAPKs and BdMAPKKs can be divided into four subgroups in A. thaliana and $O$. sativa, which were consistent with the previous report [28], whereas MAPKKKs can be subdivided into three major subtypes, Raf-like family, MEKK-like family and ZIK-like family (Additional file 2). Moreover, Raf-like family contains more than half of MAPKKKs members in all three candidate plants. In general, all MAPK cascade kinases and their subgroups were present in monocots and dicots (Additional file 2), indicating that the occurrence of most components of the MAPK cascades in plants predates the monocot-dicot divergence and MAPK cascade kinase genes were conserved during evolution. Furthermore, MAPKKK phylogenetic tree showed similar clustering patterns in O. sativa and B. distachyon. In total, about 15 pairs of MAPKs, 8 pairs of MAPKKs and 60 pairs of MAPKKKs from $O$. sativa and $B$. distachyon were clustered as pairs, indicating that they might be the orthologous genes (Additional file 5). For instance, the amino acid sequence of BdMAPKKK29 and OsMAPKKK22 showed more than eighty percent of identities, indicating many consensuses in the MAPKKK protein sequences that may have existed before the species divergence between $B$. distachyon and $O$. sativa. The phylogenetic similarity found in $O$. sativa and $B$. distachyon suggested that they might have evolved conservatively. In contrast, $B$. distachyon has less orthologous genes than those in O. sativa compared to A. thaliana and a large number of MAPK cascade kinase genes were also clustered as pairs between $B$. distachyon and $A$. thaliana, suggesting that MAPK cascade kinase genes were large conserved gene families whose origin were very old (Additional file 5). The alignment of the conserved protein kinase domains showed that all Raf-like family in $B$. distachyon as well as $O$. sativa and $A$. thaliana shared Raf-like specific polypeptide signature GTxx (W/Y) MAPE, whereas ZIK-like subfamily contained a conserved polypeptide GTPEFMAPE (L/V/M)(Y/F) and MEKK-like members shared conserved polypeptide G (T/S) Px (W/F) MAPEV (Additional file 6).

Gene duplications play an important role in biological evolution [33-35]. With the technical development of DNA sequencing, the genomic sequence data can provide substantial evidence for the abundance of duplicated genes. In order to investigate the $M A P K$ cascade kinase gene synteny among $B$. distachyon, $O$. sativa and $A$. thaliana, we searched the gene duplication within the $M A P K$, $M A P K K$ and MAPKKK gene family in these genomes of three model plants based on the information from the Plant Genome Duplication Database (http://chibba.agtec. uga.edu/duplication/index/locus). These 154 couples of duplicated genes were displayed in Additional file 7. As shown in the chromatin topology of 22 chromosomes of all genomes of three model plants duplicated segments were distributed across all 22 chromosomes, except for the most central chromosome regions, which might be the centromeric region of the chromosome (Figure 4). Generally, the distribution of orthologs along the respective chromosomes of these model plant genomes was biased. Despite the duplication of orthologs occurred in the same species, most of the gene duplications were present between B. distachyon and O. sativa (Figure 4). For example, we have found 62 pairs of gene duplication between $B$. distachyon and $O$. sativa, however, only 21 pairs of gene duplication can be found between $B$. distachyon and A. thaliana (Figure 4). These results were consistent with the current understanding of the speciation process of the three species. Furthermore, we also found that the tightly linked genes at the bottom of $B$. distachyon Chromosome 2 were still tightly linked in the $O$. sativa Chromosome 1 with the identical orientation such as the BdMAPKKK46/10/40 and OsMAPKKK31/ 40/72. A similar phenomenon could also be observed between the bottom of Chromosome 3 in $B$. distachyon and the bottom of Chromosome 2 in $O$. sativa. Interestingly, these highly similar regions were located at the low density region of $\mathrm{CpG}$ islands in both $B$. distachyon and $O$. sativa genome. Such instance could be explained as tightly linked sequences of genomic regions, which exhibited high expression, might be as donor regions in the evolution process of $B$. distachyon and $O$. sativa. The conservation of genome synteny between $B$. distachyon and $O$. sativa also confirmed that $B$. distachyon can be utilized as a temperate grass model species alternative to $O$. sativa. Moreover the evolutionary relationship between $O$. sativa and A. thaliana was remotest, which implied that $B$. distachyon might fall in between $O$. sativa and A. thaliana during evolutionary history.

\section{Expression pattern of the BdMAPK, BdMAPKK and BdMAPKKK genes in different tissues}

It has been noted previously that different members of large gene families exhibit great disparities in abundance among different tissues to accommodate different physiological processes. Thus, gene expression patterns in different tissues can provide important clues for gene functions and gene co-regulations. To extract information about the relative abundance of transcripts of $B$. distachyon MAPK cascade kinases, we tested three different tissues that were a part of $\mathrm{Bd} 21$ seedling, leaves, stems and roots, to analyze the tissue specific expression patterns of all gene family members of MAPK cascade. The expression of all of the $B d M A P K, B d M A P K K$ and BdMAPKKK gene family members was detected in all three tissues (Figure 5A-C). The results revealed that most of MAPK and MAPKK genes were highly expressed in $\mathrm{Bd} 21$ leaves, while the 


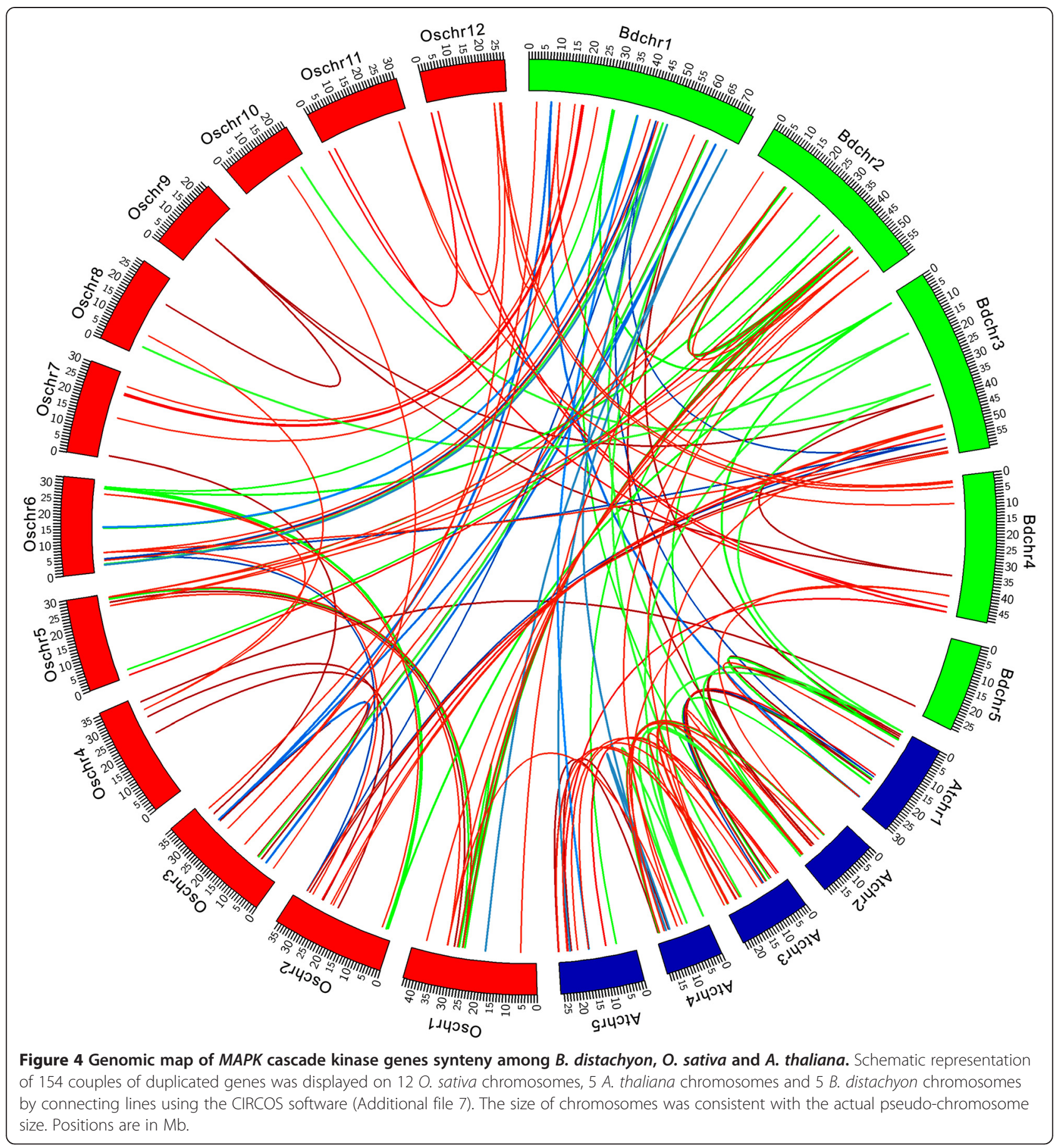

MAPKKK genes were much more abundantly expressed in the stems and leaves than that in the roots. As shown in Figure $5 \mathrm{C}$, several $B d M A P K K K$ genes, including BdMAPKKK10, -16, -37, $-44,-47$ and -49 , showed higher expression levels than other members of MAPKKK family in all of the three tissues tested. The expression of $B d M A P K K K 3,-14,-27,-34$ and -51 , was highly expressed in the leaf while its expression level was relatively low in the root and stem. The expression pattern of these genes suggested that BdMAPKKKs were involved in the growth and development of organs or tissues under specific conditions. Interestingly, the BdMKK6 exhibited a highly similar expression pattern with BdMPK6 in the three different tissues, which was consistent with the previous result that BdMKK6 exhibited a significant interaction with BdMPK6 [28]. The interacting partners, between BdMKK3-1 and BdMPK14, were also clustered together with similar expression patterns, 

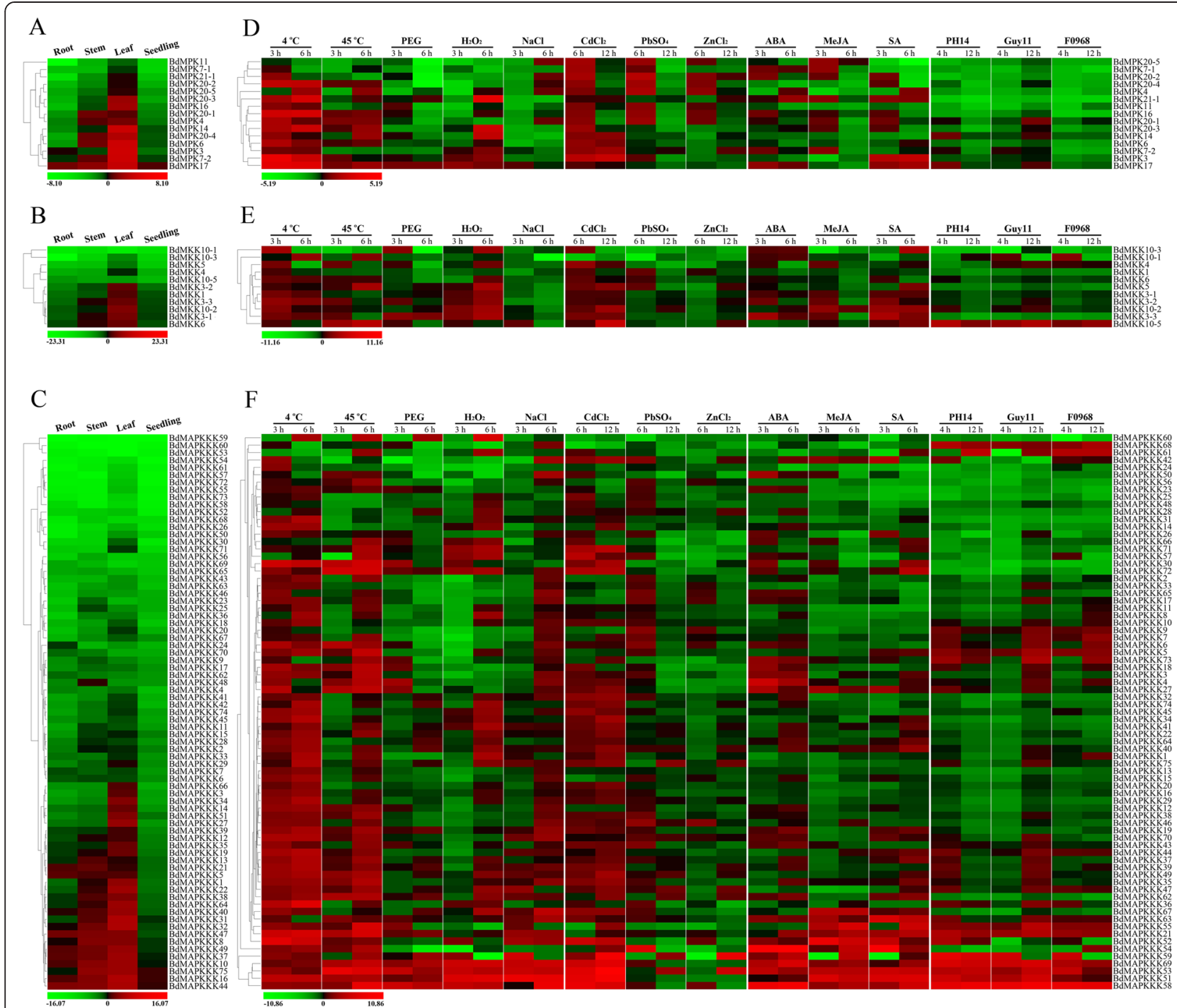

\section{F}

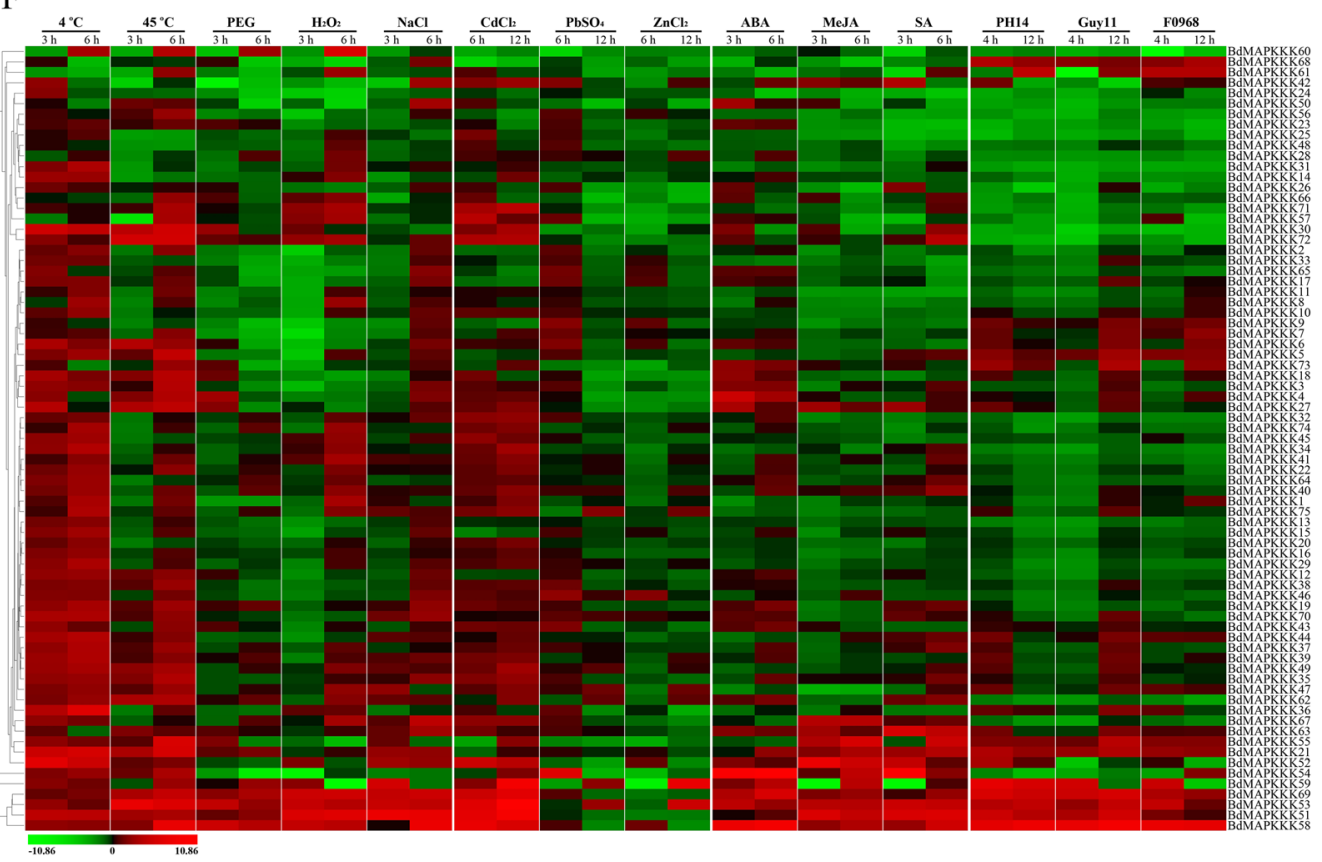

Figure 5 Expression profiles of MAPK cascade kinase genes. (A-C) Expression patterns of MAPK, MAPKK and MAPKKK genes in B. distachyon in different tissues. (D-F) Expression patterns of MAPK, MAPKK and MAPKKK genes upon multiple phytohormone treatments and abiotic or biotic stresses. The expression values of the MAPK cascade kinase genes were assessed upon the qPCR result analysis. The expression profile was shown by a green-red gradient using the PermutMatrix program.

while the expression levels of BdMPK7-1 in leaves and stems were lower than its interacting partner BdMKK31 [28]. These results suggested that the tissue specific expression patterns of all gene family members can be a possible precondition to presume possible co-regulatory genes or interacting partners.

\section{Differential expression profile of BdMAPK, BdMAPKK and} $B d M A P K K K$ gene upon multiple phytohormone treatments and abiotic or biotic stresses

In the following experiments, we demonstrated that MAPK cascades were not only involved in plant growth and development, but also played key roles in the control of plant response to multiple environmental stimuli including abiotic, biotic stresses and phytohormones. Firstly, the expression profiles of all gene members of the MAPK cascade kinases under different stress conditions were examined using qRT-PCR. A total of five abiotic stress types, i.e. heat, cold, $\mathrm{NaCl}$, PEG and $\mathrm{H} 2 \mathrm{O} 2$, which all can be activators of MAPK cascade pathway were tested in this study. Detailed expression profiles of the gene members of MAPK cascade kinases under different stress conditions were summarized in Additional file 8. Heat map representing the expression profiles of these gene members of MAPK cascade kinases under different stress conditions was shown in Figure 5D-F. Generally, our expression profile results were consistent with the Chen et al., who found that most of the MAPKs and 
MAPKKs were induced or constitutively expressed under stresses treatment [28]. Similarly, our data revealed that $90 \%$ and $60 \%$ of MAPK cascade kinase genes were up-regulated under cold and heat stress conditions, respectively. Less MAPK cascade kinase genes were up-regulated under three other kinds of abiotic stress in this study, including $\mathrm{NaCl}$, PEG and $\mathrm{H} 2 \mathrm{O} 2$, which was also consist with Chen et al., who found that only $43.75 \%$ of MAPK family genes were up-regulated in the PEG treatment [28].

These results indicated that the temperature alteration is the most sensitive stress perceived by plants. Furthermore, a large number of MAPK cascade kinase genes were unchanged or slightly down-regulated at $3 \mathrm{~h}$ after treatment with heat, $\mathrm{H}_{2} \mathrm{O}_{2}$ and $\mathrm{NaCl}$, wheaeas the genes were up-regulated at $6 \mathrm{hrs}$ after treatments. Over a half of MAPK cascade kinase genes were upregulated in both heat and cold treatments. A few MAPK cascade kinase genes were up-regulated under the conditions of all five kinds of abiotic stresses, such as BdMAPKKK51, $-53,-58,-69$ and so on. Most of the clustered gene pairs such as BdMAPKK3-1/3-2, $M A P K K K 2 / 65, M A P K K K 6 / 12$, and so on, showed the similar expression pattern after stress treatments, suggesting that these gene pairs might have similar physiological functions. On the other hand, several BdMAPKK gene pairs, which exhibited different expression patterns, may be involved in different signaling pathways. Furthermore, we also examined the expression profiles of all the gene members of MAPK cascade kinases to investigate whether the genes were involved in the response to heavy metal toxicity, including $\mathrm{CdCl}_{2}, \mathrm{PbSO}_{4}$ and $\mathrm{ZnCl}_{2}$ (Figure 5D-F). MAPK cascade kinase genes showed a very rapid increase in response to $\mathrm{CdCl}_{2}$ and $\mathrm{PbSO}_{4}$, while few MAPK cascade kinase genes were up-regulated by $\mathrm{ZnCl}_{2}$ treatment. In comparison with the abiotic stresses which have been discussed above, the expression level of $B d M A P K$ and $B d M A P K K$ genes (BdMKK1, BdMPK7-1, $B d M P K 16, B d M P K 20-1$, etc.) firstly increased within $6 \mathrm{hrs}$ after heavy metal treatment and down-regulated at 12 hrs (Figure 5C and D). The same result was obtained in the expression pattern of most of BdMAPKKK genes which was in response to $\mathrm{PbSO}_{4}$ and $\mathrm{ZnCl}_{2}$ (Figure 5F). These results suggested that MAPK cascade kinases played a crucial role in response to heavy metal stress and the response was triggered within $6 \mathrm{hrs}$ after stress treatment.

Recent studies of the MAPK cascades have also shown that the genes were responsive to JA, SA and ABA treatments. By treating plants with $\mathrm{ABA}$, several components of MAPK signaling cascade genes showed a distinct inducible expression in many plant species, suggesting an important function of MAPK pathways in ABA signaling
[36-39]. OmMKK1 was increased progressively in response to increasing lengths of exposure of MeJA, SA, ethephon and MV [40]. To investigate the hormonal control mechanisms underlying MAPK cascade kinases, we treated $\mathrm{Bd} 21$ seedlings with three phytohormones, MeJA, SA and ABA, respectively and analyzed the changes of the transcription abundance of these MAPK cascade kinase genes using qRT-PCR. Our results demonstrated that $40 \%$ of MAPK cascade kinase genes were up-regulated by these three phytohormones after $3 \mathrm{hrs}$ of treatment, respectively (Figure 5D-F). Only three of MAPK and MAPKK genes were up-regulated by all three phytohormones, whereas more than ten MAPKKKs were induced by MeJA, SA and ABA. It has reported that $\mathrm{ABA}$ as a phytohormone played an important role in integrating various abiotic stress signals and controlling downstream stress responses [41]. Our data indicated that most BdMAPKKKs were showed a similar expression pattern under drough (PEG) and $\mathrm{H}_{2} \mathrm{O}_{2}$ stress conditions compared with ABA treatment, except for BdMAPKKK3, $-4,-18,-27$ and -73 . These correlations of BdMAPKKKs expression levels between abiotic stress and phytohormone treatment suggested that the stress induced MAPK cascade signal transduction might be linked to the stress induced phytohormone alteration.

It has been reported that pathogen-associated molecular patterns (PAMPs)-triggered immunity requires a signal transduction from receptors to downstream components via the MAPK cascade, suggesting that plant MAPK cascades play a key role in the induction of defense mechanisms $[11,15,42]$. For example, the $A$. thaliana fls2 mutant was more susceptible than the wild type (WT) to infection by the virulent pathogen DC3000, and the WT showed an enhanced resistance to DC3000 after flg22 treatment [43]. To investigate the mechanisms of MAPK cascades in disease defense, we determined the expression profiles of MAPK cascade kinase genes in B. distachyon after phytopathogen treatments. A total of three phytopathogens, including Fusarium graminearum (F0968) and two strains of Magnaporthe grisea (Guy11, avirulent ACE1 genotype; PH14, virulent ACE1 genotype) were used to inoculate $\mathrm{Bd} 21$ seedling in this study. The expression profiles of the MAPK cascade kinase genes at $4 \mathrm{hpi}$ (hour post-inoculation) and 12 hpi were shown in Figure 5D-F. Only a few members of BdMAPK and BdMAPKK genes were up-regulated by phytopathogen treatment, whereas approximately $40 \%$ of BdMAPKKK genes were phytopathogen-induced. Interestingly, a large number of BdMAPKKK genes were induced faster by PH14 than by Guy11. For example, $B d M A P K K K 39,-47,-49$ and -63 were up-regulated at 4 hpi after infection by PH14, but at $12 \mathrm{hpi}$, they were down regulated by $\mathrm{PH} 14$ and up-regulated by Guy11. These 
results were consistent with the previous report about the expression pattern of $B d W R K Y$ genes, suggesting that BdMAPKKK genes might play an important role with $B d W R K Y \mathrm{~s}$ in plant defense [32]. The relationship between MAPK cascade kinases and WRKY transcript factors in phytopathogen-induced plant disease defense in $B$. distachyon should be further investigated.

\section{Regulatory network of MAPK cascade kinase genes}

In general, MAPK cascade kinase genes formed conserved signaling modules, which contained three functionally linked protein kinases: MAPKs, MAPKKs and MAPKKKs [2,3]. In this study, we constructed the regulatory network of MAPK cascade kinases upon different stress treatments in $B$. distachyon. To investigate clusters of co-expressed MAPK cascade kinase genes in B. distach$y o n$, the expression data of BdMAPKs, BdMAPKKs and $B d M A P K K K \mathrm{~s}$ were clustered together to form integrated expression profiles. In order to investigate whether the expression profiles of MAPK cascade kinase genes in a set of MAPK cascades were correlated, we compared their tissue-specific expression patterns and changes of the expression levels under different treatment conditions, including biotic stress, abiotic heavy metal stress and hormone treatment, respectively. The results showed several groups of co-expression regulatory MAPK cascade kinases whose correlation coefficients were greater than 0.5 , according to the clustered results of the integrated expression profiles (Additional file 9). The integrated expression profiles indicated that specific clusters of co-expressed MAPK cascade kinase genes were involved in response to the stresses. A scheme representing the coexpression regulatory network was constructed according to the data of co-expression regulatory MAPK cascade kinase genes (Figure 6). In A. thaliana, modules including the MAPKs MPK3/MPK6 and MAPKKs MKK1/MKK4/ MKK5/MKK9 were clearly proved to be involved in multiple stress-response strategies [11]. Consistently, those orthologous genes in $B$. distachyon, such as MAPKs BdMPK6/BdMPK7 and MAPKKs BdMKK1/BdMKK4/ BdMKK5/BdMKK6, were also found to be involved in several different types of stress treatments (Figure 6). From the scheme in Figure 6, the comparison results indicated that most of duplicated paralog pairs at the terminal nodes of the phylogenetic tree may have maintained their ancestral function. For example, two duplicated genes of BdMKK3-1/BdMKK3-2 in B. distachyon that were clustered together in the phylogenetic tree were constitutively expressed in all the tissues. These two genes were also clustered together in tissue specific expression profile. Therefore, these two duplicated genes could be classified together in the co-expression regulatory network. As shown in Figure 6, expression level changes of BdMKK6 and BdMPK6 were clustered together, in which two proteins have been proved to exhibit significant interaction with each other [28]. Moreover, some duplicated paralogous genes showed divergent function in the evolutionary process, even though the reasons for expression

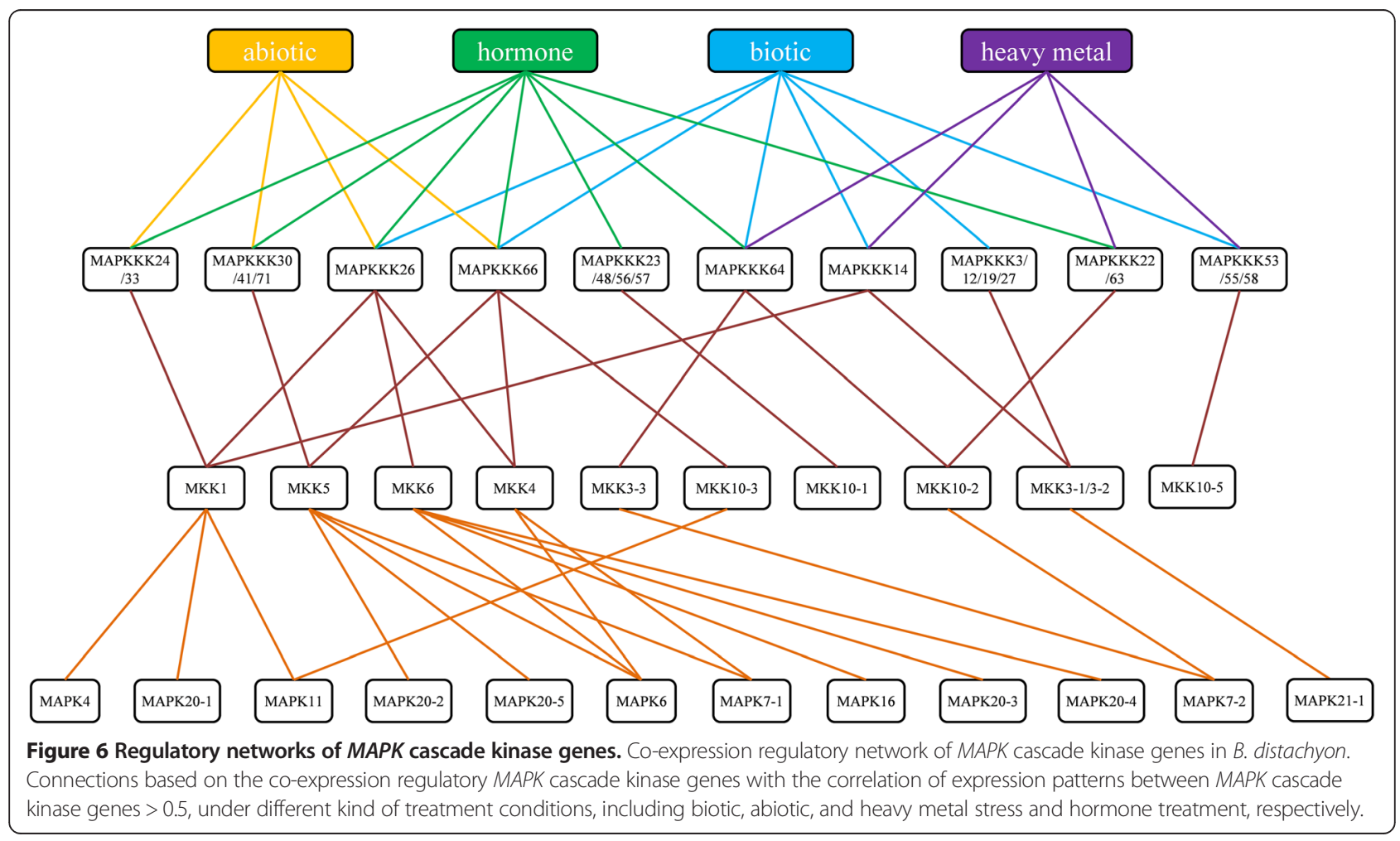


divergence of the duplicated genes in many gene families remain unclear. It has been shown that BdMPKK3-1 exhibited a significant interaction with BdMPK7-1. However, the changes of the expression level between BdMPKK3-1 and BdMPK7-1 couldn't be clustered together, while BdMPKK3-1 showed a similar expression pattern with BdMPK7-2, a homolog of BdMPK7-1. These results indicated that the scheme of the regulatory network could provide clues for a possible signaling pathway of BdMAPK cascade kinases, which show the same function or interaction in response to corresponding stress treatment. In addition, by comparing the regulatory network of MAPK cascade kinase orthologous genes in A. thaliana, our network showed a reliable result in BdMAPK signaling pathway prediction.

\section{Conclusions}

The identification and characterization of all MAPK cascade kinase genes in a grass model plant $B$ distachyon would facilitate a better understanding of the evolutionary processes and functions of these gene families. First, this study has completed identification of MAPKKK genes, the biggest upstream kinase gene family of the MAPK cascade in B. distachyon. Subsequently, this study has done systematic molecular evolutionary analysis of all MAPK, MAPKK and MAPKKK genes as a whole in $B$. distachyon comparing to $O$. sativa and A. thaliana. Furthermore, most importantly, this study has established a MAPK signaling co-regulation network to investigate the multiple-stress-driven interactions between BdMAPKs, BdMAPKKs and BdMAPKKKs upon different stresses treatment. Base on the regulation network, we found that most of duplicated BdMAPK cascade kinase gene orthologs showed their convergent function, whereas few of them developed divergent function in the evolutionary process. The molecular evolutionary analysis of identified MAPK family genes and the constructed MAPK cascade regulation network under multiple stresses provided useful information for further investigation of the functions of BdMAPK cascade kinase genes across different plant species.

\section{Methods}

\section{Sequence retrieval}

To identify $B$. distachyon genes encoding MAPKKK proteins with a kinase domain, we performed a BLASTP search among 32255 sequences of the protein database of $B$. distachyon from MIPS (http://mips.helmholtzmuenchen.de/plant/brachypodium/) using 155 query $M A P K K K$ sequences from $A$. thaliana and $O$. sativa [1]. A self BLAST of these sequences followed by manual editing to remove the redundancy finally resulted in the identification of 75 MAPKKK genes. To verify the reliability of our results, all putative non-redundant sequences were assessed with UniProt (http://www.uniprot.org/) and SMART (http://smart.embl-heidelberg. de/) analysis, respectively. A total of 75 BdMAPKKK genes were found in $B$. distachyon (Additional file 1). All of these 75 putative $M A P K K K$ gene family members in $B$. distachyon genome were designed as BdMAPKKK1$B d M A P K K K 75$ base on the BBMH scores between putative $B d M A P K K K$ genes with MAPKKK gene family in $O$ sativa. The annotation of 16 MAPK genes and 12 MAPKK genes from $B$. distachyon $\mathrm{Bd} 21$ genome in Additional file 1 were made according to the previous research [28].

\section{Sequence and phylogenetic analysis}

To analyze the sequence of the 75 typical identified BdMAPKKKs, 12 MAPKKs and 16 MAPKs, we performed multiple alignment analyses of the kinase domains, sequence by ClustalW (www.ebi.ac.uk/clustalw/). A neighbor-joining (NJ) tree was constructed using the MEGA version 5 software, based on the alignment of MAPK cascade nucleotide or amino acid sequences in $O$. sativa, A. thaliana and $B$. distachyon. To determine the statistical reliability, we conducted bootstrap analysis with the following parameters: $\mathrm{p}$-distance and pairwise deletion. Bootstrap analysis was performed with 1000 replicates. The data of the phylogenetic tree were deposited in Treebase Web (Accession URL: http://www. psort.org/).

\section{Gene structure analysis}

The information of BdMAPK cascade kinase genes, including accession number, chromosomal location, ORF length and exon-intron structure, were retrieved from the $B$. distachyon genome Database (http://www.brachypodium.org/). As well as the gene struc-tures of the BdMAPK cascade kinases were generated with the GSDS version 2.0 (Gene Structure Display Server 2: http://gsds. cbi.pku.edu.cn/).

\section{Protein analysis of BdMAPK cascade kinases}

The conserved domains and motifs in the MAPK cascade kinases was predicted using InterProScan against protein databases (http://www.ebi.ac.uk/interpro/). The schematic representing the structure of all members of BdMAPK cascade kinases was based on the InterProScan analysis results. Subcellular localization predictions of each of the BdMAPK cascade kinases were carried out using WoLF PSORT server (http://purl.org/phylo/treebase/phylows/study/ TB2:S17106? $x$-accesscode $=37158 a 103 e 4 a c a e a 162 c 89867 a 5 c 7$ 061\&format=html). The theoretical pI (isoelectric point) and $\mathrm{Mw}$ (molecular weight) of BdMAPK cascade kinases were carried out using Compute pI/Mw tool online (http://web. expasy.org/compute_pi/). Subcellular localization predictions 
of each of the BdMAPK cascade kinases were carried out using WoLF PSORT server (http://wolfpsort.seq.cbrc.jp/).

\section{MAPKKK gene synteny analysis}

The gene duplications within the MAPK cascade kinase gene family in $B$. distachyon, $O$. sativa and $A$. thaliana genomes were based on the information from the Plant Genome Duplication Database (http://chibba.agtec.uga. edu/duplication/index/locus). In order to visualize duplicated regions in the $B$. distachyon, $O$. sativa and $A$. thaliana genome, lines were drawn between matching genes using Circos-0.64 program (http://circos.ca/) [44].

\section{Cluster analysis of expression data}

The 2-week old seedlings (Bd21) were used for harvesting leaf, stem and root samples. The protocol of abiotic stresses treatment for Bd21 was adopted according with the previous work with some modifies [28]. For phytohormone analysis, 2-week-old seedlings were treated in MS liquid medium containing $100 \mu \mathrm{M}$ MeJA, $100 \mu \mathrm{M}$ $\mathrm{ABA}, 1 \mathrm{mM} \mathrm{SA}$ and $20 \mu \mathrm{M}$ 6-BA for $3 \mathrm{~h}$ or $6 \mathrm{~h}$, respectively. For abiotic stress treatment, 2-week-old seedlings were treated in MS liquid medium containing 20\% PEG, $200 \mathrm{mM} \mathrm{NaCl}$ and $10 \mathrm{mM} \mathrm{H}_{2} \mathrm{O}_{2}$ for $3 \mathrm{~h}$ or $6 \mathrm{~h}$, respectively. For heavy metal stress treatment, 2-week-old seedlings were treated in MS liquid medium containing $100 \mu \mathrm{M} \mathrm{ZnCl}, 100 \mu \mathrm{M} \mathrm{PbSO}_{4}$ and $100 \mu \mathrm{M} \mathrm{CdCl}_{2}$ for $6 \mathrm{~h}$ or $12 \mathrm{~h}$, respectively. Cold and heat treatments were achieved by placing 2-week-old seedlings in MS liquid medium at $4^{\circ} \mathrm{C}$ or $45^{\circ} \mathrm{C}$ for $3 \mathrm{~h}$ or $6 \mathrm{~h}$, respectively. For phytopathogen treatment, 2-week-old seedlings were sprayed with Fusarium graminearum (F0968) and two strains of Magnaporthe grisea (Guy11, avirulent ACE1 genotype; PH14, virulent ACE1 genotype) for $4 \mathrm{~h}$ or $12 \mathrm{~h}$. The samples of each treatment were collected three biological replications. The BdMAPK cascade kinase genes array constituted of 103 primer-sets representing all members of the $B$. distachyon MAPK cascade kinase gene family. The primer-sets were listed in Additional file 10 . The expression of the 103 BdMAPK cascade kinase genes was assessed upon the qPCR result analysis. Each qPCR experiment was repeated three separate times. The expression profile was calculated from the $-\Delta \Delta C \mathrm{~T}$ value $[-\Delta \Delta C \mathrm{~T}=(C$ Tcontrol.gene $-C$ Tcontrol.actin $)-$ (CTtreat.gene $-C$ Ttreat.actin)], obtained by PermutMatrixEN version 1.9.3 software, and shown by a green-red gradient. The data were statistically analyzed using OriginPro 7.5 software. The up-regulated genes were defined as a fold-change greater than 2 with $p$-value $<0.05$ and a fold change of 0.5 or less was used to define downregulated genes when the $p$-value $<0.05$. All qPCR data were submitted to NCBI GEO dataset. The accession number is GSE66497.

\section{Regulatory network construction}

The expression data of $B d M A P K, B d M A P K K$ and $B d M A P K K K$ were clustered together to form an integrated expression profile by Cluster 3.0 software and visualized by using TreeView software. The MAPK cascade kinase genes, whose correlation coefficients of expression profiles were greater than 0.5 , were clustered together as a set of co-expression regulatory MAPK cascade kinase genes under different kinds of treatment conditions, including biotic, abiotic, heavy metal stress and hormone treatment, respectively (Additional file 9). The line drawing which represented the co-expression regulatory network was constructed according to the data of co-expression regulatory $M A P K$ cascade kinase genes.

\section{Additional files}

Additional file 1: List of MAPK cascade kinase genes in B. distachyon. Additional file 2: Neighbor-joining analyses of MAPK (A), MAPKK (B) and MAPKKK (C) amino acid sequences from $O$. sativa, $A$. thaliana and B. distachyon.

Additional file 3: List of MAPK cascade kinase genes in O. sativa, A. thaliana and $B$. distachyon chromosomes.

Additional file 4: The amino acid sequences of MAPKKK members in B. distachyon, A. thaliana and O. sativa.

Additional file 5: Orthologs of MAPK cascade kinase genes among B. distachyon, A. thaliana and $O$. sativa.

Additional file 6: Alignment of MAPKKKs from O. sativa, A. thaliana and $B$. distachyon.

Additional file 7: The duplicated genes in O. sativa, A. thaliana and B. distachyon chromosomes.

Additional file 8: Expression data of the BdMAPK cascade kinase genes after stresses and phytohormone treatment.

Additional file 9: The co-expression regulatory MAPK cascade kinase genes.

Additional file 10: The list of qRT-PCR primers of MAPK cascade kinase genes.

\section{Competing interests}

The authors declare that they have no competing interests.

\section{Authors' contributions}

ZC designed and coordinated the work. MJ and FW performed most of the data mining and data analysis. MJ performed the GPCR experiment, FW and ZC wrote the manuscript. JC, PL and JS helped to retrieve gene sequences data. All authors read and approved the final manuscript.

\section{Acknowledgements}

We want to thank the contributors of the B. distachyon Genome Database, which was a convenient tool used to search for BdMAPKKK genes. This work was supported by Grant for Starting Package to Research Group of Plant Abiotic Stress and Plant Genome Evolution in Shanghai Chenshan Plant Science Research Centre, Chinese Academy of Sciences and Shanghai Chenshan Botanic Garden from Shanghai Landscaping Administrative Bureau (NO. F0112423, F0122415, F122423, F132426) and the Fund for National Key Laboratory of Plant Molecular Genetics (Y109Z11161).

\section{Author details}

'Shanghai Chenshan Plant Science Research Center, Shanghai Chenshan Botanical Garden, Shanghai Key Laboratory of Plant Functional Genomics and Resources, Shanghai Institutes for Biological Sciences, Chinese Academy of Sciences, 3888 Chenhua Road, 201602 Shanghai, Songjiang, China. 
${ }^{2}$ Current address: College of Pharmacy and Life Science, Jiujiang University, Jiujiang 332000, China. ${ }^{3}$ Program of Biochemistry and Immunology, University of Toronto, 100 St. George Street, Toronto M5G 3G3, Ontario, Canada.

\section{Received: 28 July 2014 Accepted: 9 March 2015} Published online: 24 March 2015

\section{References}

1. International Brachypodium I. Genome sequencing and analysis of the model grass Brachypodium distachyon. Nature. 2010;463(7282):763-8.

2. Tena G, Asai T, Chiu WL, Sheen J. Plant mitogen-activated protein kinase signaling cascades. Curr Opin Plant Biol. 2001;4(5):392-400.

3. Mishra NS, Tuteja R, Tuteja N. Signaling through MAP kinase networks in plants. Arch Biochem Biophys. 2006;452(1):55-68.

4. Rodriguez MC, Petersen M, Mundy J. Mitogen-activated protein kinase signaling in plants. Annu Rev Plant Biol. 2010;61:621-49.

5. Nakagami H, Soukupova H, Schikora A, Zarsky V, Hirt H. A Mitogen-activated protein kinase kinase kinase mediates reactive oxygen species homeostasis in Arabidopsis. J Biol Chem. 2006;281(50):38697-704.

6. Suarez-Rodriguez MC, Adams-Phillips L, Liu Y, Wang H, Su SH, Jester PJ, et al. MEKK1 is required for flg22-induced MPK4 activation in Arabidopsis plants. Plant Physiol. 2007;143(2):661-9.

7. Asai T, Tena G, Plotnikova J, Willmann MR, Chiu WL, Gomez-Gomez L, et al. MAP kinase signalling cascade in Arabidopsis innate immunity. Nature. 2002;415(6875):977-83

8. Takahashi F, Yoshida R, Ichimura K, Mizoguchi T, Seo S, Yonezawa M, et al. The mitogen-activated protein kinase cascade MKK3-MPK6 is an important part of the jasmonate signal transduction pathway in Arabidopsis. Plant Cell. 2007;19(3):805-18.

9. Yue H, Li Z, Xing D. Roles of Arabidopsis bax inhibitor-1 in delaying methyl jasmonate-induced leaf senescence. Plant Signal Behav. 2012;7(11):1488-9.

10. Ichimura K, Mizoguchi T, Irie K, Morris P, Giraudat J, Matsumoto K, et al. Isolation of ATMEKK1 (a MAP kinase kinase kinase)-interacting proteins and analysis of a MAP kinase cascade in Arabidopsis. Biochem Biophys Res Commun. 1998;253(2):532-43.

11. Pitzschke A, Schikora A, Hirt H. MAPK cascade signalling networks in plant defence. Curr Opin Plant Biol. 2009;12(4):421-6.

12. Group M. Mitogen-activated protein kinase cascades in plants: a new nomenclature. Trends Plant Sci. 2002;7(7):301-8.

13. Kiegerl S, Cardinale F, Siligan C, Gross A, Baudouin E, Liwosz A, et al. SIMKK a mitogen-activated protein kinase (MAPK) kinase, is a specific activator of the salt stress-induced MAPK, SIMK. Plant Cell. 2000;12(11):2247-58.

14. Seo S, Katou S, Seto H, Gomi K, Ohashi Y. The mitogen-activated protein kinases WIPK and SIPK regulate the levels of jasmonic and salicylic acids in wounded tobacco plants. Plant J. 2007:49(5):899-909.

15. Desikan R, Hancock JT, Ichimura K, Shinozaki K, Neill SJ. Harpin induces activation of the Arabidopsis mitogen-activated protein kinases AtMPK4 and AtMPK6. Plant Physiol. 2001;126(4):1579-87.

16. Hadiarto T, Nanmori T, Matsuoka D, Iwasaki T, Sato K, Fukami $Y$, et al. Activation of Arabidopsis MAPK kinase kinase (AtMEKK1) and induction of AtMEKK1-AtMEK1 pathway by wounding. Planta. 2006;223(4):708-13.

17. Teige $M$, Scheikl E, Eulgem T, Doczi R, Ichimura K, Shinozaki K, et al. The MKK2 pathway mediates cold and salt stress signaling in Arabidopsis. Mol Cell. 2004;15(1):141-52.

18. Pitzschke A, Djamei A, Bitton F, Hirt H. A major role of the MEKK1-MKK1/2-MPK4 pathway in ROS signalling. Mol Plant. 2009;2(1):120-37.

19. Wen JQ, Oono K, Imai R. Two novel mitogen-activated protein signaling components, OsMEK1 and OsMAP1, are involved in a moderate low-temperature signaling pathway in rice. Plant Physiol. 2002;129(4):1880-91.

20. Champion A, Picaud A, Henry Y. Reassessing the MAP3K and MAP4K relationships. Trends Plant Sci. 2004;9(3):123-9.

21. Jonak C, Okresz L, Bogre L, Hirt H. Complexity, cross talk and integration of plant MAP kinase signalling. Curr Opin Plant Biol. 2002;5(5):415-24.

22. Kong X, Lv W, Zhang D, Jiang S, Zhang S, Li D. Genome-wide identification and analysis of expression profiles of maize mitogen-activated protein kinase kinase kinase. PLoS One. 2013;8(2):e57714.

23. Kieber JJ, Rothenberg M, Roman G, Feldmann KA, Ecker JR. CTR1, a negative regulator of the ethylene response pathway in Arabidopsis, encodes a member of the raf family of protein kinases. Cell. 1993;72(3):427-41.
24. Huang Y, Li H, Hutchison CE, Laskey J, Kieber JJ. Biochemical and functional analysis of CTR1, a protein kinase that negatively regulates ethylene signaling in Arabidopsis. Plant J. 2003;33(2):221-33.

25. Frye CA, Tang D, Innes RW. Negative regulation of defense responses in plants by a conserved MAPKK kinase. Proc Natl Acad Sci U S A. 2001;98(1):373-8.

26. Frye CA, Innes RW. An Arabidopsis mutant with enhanced resistance to powdery mildew. Plant Cell. 1998;10(6):947-56.

27. Xu BE, Lee BH, Min X, Lenertz L, Heise CJ, Stippec S, et al. WNK1: analysis of protein kinase structure, downstream targets, and potential roles in hypertension. Cell Res. 2005;15(1):6-10.

28. Chen L, Hu W, Tan S, Wang M, Ma Z, Zhou S, et al. Genome-wide identification and analysis of MAPK and MAPKK gene families in Brachypodium distachyon. PLoS One. 2012;7(10):e46744.

29. Rao KP, Richa T, Kumar K, Raghuram B, Sinha AK. In silico analysis reveals 75 members of mitogen-activated protein kinase kinase kinase gene family in rice. DNA Res. 2010;17(3):139-53.

30. Yin Z, Wang J, Wang D, Fan W, Wang $S$, Ye W. The MAPKKK gene family in Gossypium raimondii: genome-wide identification, classification and expression analysis. Int J Mol Sci. 2013;14(9):18740-57.

31. Taj G, Agarwal P, Grant M, Kumar A. MAPK machinery in plants: recognition and response to different stresses through multiple signal transduction pathways. Plant Signal Behav. 2010;5(11):1370-8.

32. Wen F, Zhu H, Li P, Jiang M, Mao W, Ong C, et al. Genome-wide evolutionary characterization and expression analyses of WRKY family genes in brachypodium distachyon. DNA Res. 2014;21(3):327-39.

33. Ohta T. Evolution by gene duplication and compensatory advantageous mutations. Genetics. 1988;120(3):841-7.

34. Ohta T. Role of gene duplication in evolution. Genome. 1989;31(1):304-10

35. Tang J, James MN, Hsu IN, Jenkins JA, Blundell TL. Structural evidence for gene duplication in the evolution of the acid proteases. Nature. 1978;271(5646):618-21

36. Zhang M, Pan J, Kong X, Zhou Y, Liu Y, Sun L, et al. ZmMKK3, a novel maize group B mitogen-activated protein kinase kinase gene, mediates osmotic stress and ABA signal responses. J Plant Physiol. 2012;169(15):1501-10.

37. Jammes F, Song C, Shin D, Munemasa S, Takeda K, Gu D, et al. MAP kinases MPK9 and MPK12 are preferentially expressed in guard cells and positively regulate ROS-mediated ABA signaling. Proc Natl Acad Sci U S A. 2009;106(48):20520-5.

38. Zhang A, Jiang M, Zhang J, Tan M, Hu X. Mitogen-activated protein kinase is involved in abscisic acid-induced antioxidant defense and acts downstream of reactive oxygen species production in leaves of maize plants. Plant Physiol. 2006;141(2):475-87.

39. Danquah A, de Zelicourt A, Colcombet J, Hirt H. The role of ABA and MAPK signaling pathways in plant abiotic stress responses. Biotechnol Adv. 2014;32(1):40-52.

40. You MK, Oh SI, OK SH, Cho SK, Shin HY, Jeung JU, et al. Identification of putative MAPK kinases in Oryza minuta and $\mathrm{O}$. sativa responsive to biotic stresses. Mol Cells. 2007;23(1):108-14.

41. Tuteja N. Abscisic Acid and abiotic stress signaling. Plant Signal Behav. 2007;2(3):135-8.

42. Droillard MJ, Boudsocq M, Barbier-Brygoo H, Lauriere C. Involvement of MPK4 in osmotic stress response pathways in cell suspensions and plantlets of Arabidopsis thaliana: activation by hypoosmolarity and negative role in hyperosmolarity tolerance. FEBS Lett. 2004;574(1-3):42-8.

43. Zipfel C, Robatzek S, Navarro L, Oakeley EJ, Jones JD, Felix G, et al. Bacterial disease resistance in Arabidopsis through flagellin perception. Nature. 2004:428(6984):764-7.

44. Krzywinski M, Schein J, Birol I, Connors J, Gascoyne R, Horsman D, et al. Circos: an information aesthetic for comparative genomics. Genome Res. 2009;19(9):1639-45. 